\title{
Assessing the impact of posture on diaphragm morphology and function using an open upright MRI system - a pilot study
}

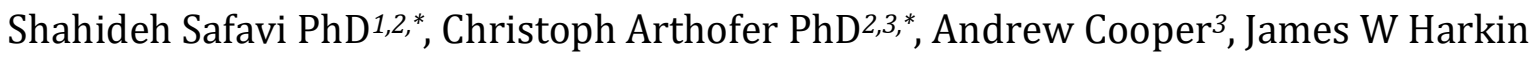
MPhys ${ }^{1}$, Andrew P Prayle PhD ${ }^{4}$, Milind P Sovani MD ${ }^{5}$, Charlotte E Bolton PhD ${ }^{2,6}$, Penny A Gowland $\mathrm{PhD}^{3}$, Ian $\mathrm{P}$ Hall $\mathrm{PhD}^{1,2}$

1- Respiratory Medicine Department, School of Medicine, University of Nottingham, Queen's Medical Centre Campus, Nottingham, UK.

2- NIHR Nottingham Biomedical Research Centre, Queen's Medical Centre, Nottingham NG7 2UH, UK

3- Sir Peter Mansfield Imaging Centre, University of Nottingham, University of Nottingham, University Park, Nottingham, UK.

4- Paediatric Respiratory Medicine Department, Queen's Medical Centre, Nottingham, UK

5- Respiratory Medicine Department, Queen's Medical Centre, Nottingham University Hospitals NHS Trust, Nottingham, UK

6- Respiratory Medicine, School of Medicine, University of Nottingham, Nottingham City Hospital Campus, Hucknall Road, Nottingham, UK.

*Shahideh Safavi and Christoph Arthofer are joint first authors.

\section{Corresponding Author: Dr Shahideh Safavi}

Respiratory Medicine Department, School of Medicine, University of Nottingham, Queen's Medical Centre Campus, Nottingham NG7 2UH, United Kingdom

Phone: +44 1158231154

Email: shahideh.safavi@nottingham.ac.uk

Funding: This research was funded by the University of Nottingham and supported by the NIHR Nottingham Biomedical Research Centre. 


\section{Summary conflict of interest statements}

SS, AC, JWH, MPS, CEB, and PG have no conflicts of interest.

APP reports grants from Thrasher Research Fund, grants from Action for Ataxia Telangiectasia, both supporting work to develop MRI imaging of the lungs in children, outside the submitted work.

CA was funded by the NIHR Nottingham Biomedical Research Centre, during the conduct of the study.

IPH reports grants from NIHR, during the conduct of the study; grants from GSK, grants from $\mathrm{BI}$, outside the submitted work. 


\title{
Assessing the impact of posture on diaphragm morphology and function using an open upright MRI system - a pilot study
}

\begin{abstract}
Purpose: The diaphragm is the most important muscle of respiration. Disorders of the diaphragm can have a deleterious impact on respiratory function. We aimed to evaluate the use of an open-configuration upright low-field MRI system to assess diaphragm morphology and function in patients with bilateral diaphragm weakness (BDW) and chronic obstructive pulmonary disease (COPD) with hyperinflation.
\end{abstract}

Method: The study was approved by the National Research Ethics Committee, and written consent was obtained. We recruited 20 healthy adult volunteers, six subjects with BDW, and five subjects with COPD with hyperinflation. We measured their vital capacity in the upright and supine position, after which they were scanned on the 0.5 T MRI system during 10-second breath-holds at end-expiration and end-inspiration in both positions. We developed and applied image analysis methods to measure the volume under the dome, maximum excursion of hemidiaphragms, and anterior-posterior and left-right extension of the diaphragm.

Results: All participants were able to complete the scanning protocol. The patients found scanning in the upright position more comfortable than the supine position. All differences in the supine inspiratory-expiratory parameters, excluding left-right extension, were significantly smaller in the BDW and COPD groups compared with healthy volunteers. No significant correlation was found between the postural change in diaphragm morphology and vital capacity in either group.

Conclusion: Our combined upright-supine MR imaging approach facilitates the assessment of the impact of posture on diaphragm morphology and function in patients with BDW and those with COPD with hyperinflation. 


\section{KEYWORD}

upright MRI, diaphragm, bilateral diaphragm weakness, COPD

\section{ABBREVIATIONS}

$\begin{array}{ll}\text { ANOVA } & \text { Analysis of variance } \\ \text { BDW } & \text { bilateral diaphragm weakness } \\ \text { COPD } & \text { chronic obstructive pulmonary disease } \\ \text { CT } & \text { Computed tomography } \\ \text { MRC } & \text { Medical Research Council } \\ \text { MRI } & \text { Magnetic resonance imaging } \\ \text { NNI } & \text { natural neighbour interpolation } \\ \text { SGRQ } & \text { St George's Respiratory Questionnaire } \\ \text { VuD } & \text { volume under the dome }\end{array}$




\section{INTRODUCTION}

The diaphragm is the most important muscle of respiration $(1,2)$. Its disorders can have a significant deleterious impact on respiratory function. The clinical manifestation is often insidious and dependent on the underlying aetiology and disease severity (3). Patients with severe bilateral diaphragm weakness (BDW) frequently present with shortness of breath on exertion, breathlessness on lying supine, and disturbed sleep $(4,5)$.

Diaphragm function can also be impaired in a range of other respiratory diseases, including chronic obstructive pulmonary disease (COPD) with chronic hyperinflation, where diaphragm fibres are shortened, impairing its force generating-capacity $(6,7)$. A decrease in the diaphragm curvature and the zone of apposition, coupled with the change in the mechanical arrangement of diaphragm fibres, contribute to compromised diaphragmatic function (6).

A range of approaches have been used to assess diaphragm function, although none optimally address both structure and function. Pulmonary function tests, particularly measurement of vital capacity in upright and supine positions, are the most commonly used first-line diagnostic tool $(3,8)$ but provide no anatomical information. Videofluoroscopy can provide dynamic functional information (8). However, the breathing pattern changes in BDW and pathology may be missed (9). Additionally, videofluoroscopy is effort-dependent (1).

Ultrasound is increasingly used to assess the dynamic change in diaphragm thickness with respiration $(3,8)$, but it is relatively low resolution. As the diaphragm is poorly echogenic, its identification is dependent on bright echoes reflected from the attached parietal pleural and peritoneal membranes, and the underlying organs. Hence, visualising the right hemidiaphragm overlying the liver is easier than visualising the left hemidiaphragm that is adjacent to the gastric and intestinal gas (8). The diaphragm borders are poorly defined on ultrasound; hence, quantitative volumetric assessments of diaphragm excursion are relatively inaccurate (8). Additionally, ultrasound images are 
operator dependent: the position and angulation of the transducer is crucial and can be challenging to maintain during the respiratory cycle, risking poor visualisation.

Computed tomography (CT) is the primary cross-sectional imaging modality used in investigating the aetiology of BDW (9). However, conventional CT requires the subject to lie down in the scanner, which can be problematic in those with diaphragm weakness. Additionally, CT scans involve exposure to ionising radiation.

Given the above limitations, there has been an interest in the use of magnetic resonance imaging (MRI) for diaphragm imaging (10-12), particularly as MRI is operatorindependent and allows three-dimensional characterisation of morphological changes of the whole diaphragm. However, many patients with respiratory disease will have difficulty with the need to lie flat and some may find even wide-bore MRI scanners claustrophobic. Hence, conventional MRI is not used routinely in clinical settings to assess patients with potentially abnormal diaphragm function.

This pilot study aimed to assess the feasibility an open-configuration upright low-field MRI system to compare diaphragm morphology and function during the breathing cycle in sitting and supine positions in healthy volunteers and subjects with BDW and COPD with hyperinflation. Furthermore, the difference between different disease pathologies has been evaluated. 


\section{METHODS}

Upright MRI was used to measure changes in diaphragm morphology during the breathing cycle, in both supine and seated positions in healthy volunteers and subjects with BDW and COPD.

\section{Study subjects}

We obtained ethical approval from the National Research Ethics Committee (reference 18/WA/0148). The trial is registered with Clinical Trials.Gov (NCT03531775). Participants provided written consent before recruitment.

The study was conducted from July to September 2018. General inclusion criteria were aged 18 years and over and the ability to provide informed consent, lie flat, and perform a 10-second breath-hold. The specific criteria for the patients included the prior diagnosis of BDW or COPD with hyperinflation (as evidenced by pulmonary function test or plain chest radiograph (CXR) by a respiratory physician. General exclusion criteria were standard contraindications to undergoing MRI and inability to fit in the scanner and/or coil due to body habitus. Healthy participants were excluded if they had a history of chronic respiratory disease and/or significant smoking history (defined as more than 10pack-years). History of lung volume reduction procedure was an exclusion criterion for the COPD group. Three individuals were excluded at the screening stage: one did not meet the MRI safety criteria and two did not fit in the coil. We subsequently recruited twenty healthy participants via advertisement, and six subjects with BDW and five with COPD with hyperinflation from a tertiary respiratory centre.

In clinical settings, hyperinflation may be diagnosed on either pulmonary function tests or CXR. Residual volume (RV), functional residual capacity (FRC), and total lung capacity (TLC) are the PFT parameters used to diagnose lung hyperinflation. Hyperinflation is diagnosed when these parameters exceed either the upper limit of normal or an arbitrary $120 \%$ of the predicted value (13). It may be diagnosed on CXR if more than six anterior ribs or 10 posterior ribs are visible on the midclavicular line. In hyperinflation, the dome of the diaphragm is typically seen below the seventh rib (14), and the lung fields are hyperlucent. The participants had pulmonary function testing and CXR as part of their 
clinical care. Those results, within six months of recruitment to the study, have been used in this paper.

Vital capacity was measured in upright and supine positions, using the CareFusion MicroLab Spirometer (CareFusion UK 232 Ltd, Quayside, UK), on the scan day.

All subjects filled the St George's Respiratory Questionnaire (SGRQ) and Medical Research Council (MRC) dyspnoea score before scanning. They also filled a visual analogue breathlessness scale following the completion of scanning to report how easy or difficult they found breathing in the scanner in the two positions (scale 1-10, with $1=$ very easy and 10 = very difficult).

\section{Image acquisition}

We scanned the subjects in supine and upright (seated) positions at end-inspiration and end-expiration on an open-configuration upright 0.5T MRI scanner (Paramed, Genoa, Italy) (Figure $1 \mathrm{~A}-\mathrm{B}$ ) with either a four-channel rigid or a single channel flexible body coil (both manufactured by Paramed), depending on the body habitus. We acquired three sagittal and three coronal plane scans for each of the four conditions, which included supine end-inspiration, supine end-expiration, seated end-inspiration, and seated endexpiration, resulting in a total of 24 scans. No contrast agents were used.

The sequences available on this scanner are limited by gradient interactions with the magnet. The scanner offered no gradient echo sequences capable of acquiring multiple slices within a single breath-hold. We modified a two-dimensional spin-echo T1weighted sequence to include image acquisition triggered using an external Tektronix AFG3021 function generator. This minimised the breath hold required for the scan. The scan parameters are shown in Table 1. Half Fourier acquisitions were used for both coronal and sagittal scans to reduce the number of phase-encoding steps required. The duration of the breath-hold was 10 seconds for each scan.

\section{Image analysis}


We developed a semi-automated image processing pipeline in MATLAB (MathWorks, Natick, MA, USA) to characterise changes in the diaphragm shape. Points were manually selected on the diaphragm on each slice. These scattered points were mapped on a regularly spaced grid with 2 millimetres spacing (Figure 2 A). The excursion at unsampled grid coordinates was estimated with natural neighbour interpolation (NNI). NNI is capable of estimating a smooth approximation by finding the closest input points to a query point and calculating a weighted average based on adjacency relationships and volume ratios of the Voronoi diagram respectively. The final average surface was estimated by repeatedly alternating between rigid registrations of the individual three coronal and three sagittal point clouds to the points sampled from the average surface of the previous iteration, and the estimation of a new average surface which is the surface fitted to the merged registered point clouds (Figure $2 \mathrm{~A}-\mathrm{B}$ ). Iterative closest point registration was used for aligning the point clouds.

We compared the change in each morphological parameter with the breathing cycle in seated and supine positions. Diaphragm morphology was characterised by five morphological parameters (indicated on Figure 1 C-D): volume under the dome of the diaphragm (VuD), excursion of each hemidiaphragm, and left-right and anteriorposterior extensions. VuD was defined as the volume between the diaphragm surface and a planar base surface spanning axially at the most inferior point of the diaphragm. The excursion was measured as the maximum perpendicular distance between this planar base surface and the hemidiaphragm surface in cranio-caudal direction. The extension of the diaphragm surface was measured axially in anterior-posterior and left-right directions by calculating the distance between the most lateral points, in the sagittal and coronal plane scans, respectively.

\section{Statistical analysis}

Statistical analysis was performed in SPSS (IBM Corporation, version 26.0). Paired t-test and analysis of variance (ANOVA) were used for within-group comparison of the results. Welch's t-test was used to compare the healthy volunteer group with the COPD and BDW groups. A p-value $<0.05$ was considered statistically significant for these tests. 
The Pearson correlation coefficient was used to assess whether the change in VuD during the breathing cycle (end-inspiration - end-expiration) in the seated position compared to the supine position correlated with the postural change in vital capacity with $\mathrm{p}<0.05$ considered statistically significant. $\mathrm{VuD}$ was chosen as the most representative value for comparison as it is a composite measurement of the other four parameters obtained and representative of diaphragm function. Hence, it is the best diaphragm parameter to compare with vital capacity.

To assess reproducibility, each scan (e.g. coronal plane scan at end-inspiration in seated position) was repeated three times, and ANOVA was used for statistical assessment of within-subject variability. 


\section{RESULTS}

All subjects were able to complete the 24 scans. Subject demographics, lung function parameters, and respiratory questionnaire scores are summarised in Table 2 . Representative coronal and sagittal plane images of the three groups are depicted in Figure 3.

\section{Change in diaphragm morphology}

Table 3 summarises assessment of diaphragm morphology and changes with posture and the breathing cycle, using the five morphological parameters.

In all groups, all diaphragm morphological parameters increased at end-inspiration compared to end-expiration in the seated (Table 3 and Figure 4). In the healthy volunteers, there was a statistically significant increase at end-inspiration in all parameters in both postures $(\mathrm{p}<0.0001$ for all parameters). In the BDW group, the increase in $\mathrm{VuD}$ and anterior-posterior extension at end-inspiration was statistically significant only in the supine position ( $\mathrm{p}=0.012$ and 0.011 , respectively), while a statistically significant change in left-right extension was seen in both postures. However, the excursion of the hemidiaphragms was not significantly changed with the breathing cycle at either posture. In the COPD group, in contrast, the increase in left-right extension at end-inspiration was statistically significant in both seated and supine positions ( $p=0.002$ and 0.008, respectively); however, a significant increase in the other four parameters was only seen in the seated position.

The difference in the change in the morphological parameters with the breathing cycle (end-inspiration - end-expiration) for the two postures, between the healthy volunteers and the two patient groups, are summarised in Table 4. Individual subject values are shown in Figure 3. The absolute change in each parameter with the breathing cycle was greater in the healthy volunteers compared to the BDW and COPD groups. The change in $\mathrm{VuD}$ and excursion of the right and left hemidiaphragms was significantly greater in the healthy volunteers than the BDW group only in the supine position $(p=0.0017,0.0439$, and 0.0075 , respectively), while the change in anterior-posterior extension was 
significantly greater in both postures; no significant difference between the two groups was found in the left-right extension.

The pattern of change in the COPD group was different from that seen in the BDW group. The change in $\mathrm{VuD}$ and excursion of the right and left hemidiaphragms was significantly greater in the supine position for the healthy volunteers compared to the COPD group $(\mathrm{p}=0.0125,0.0479$, and 0.0281 , respectively), whilst the change in left-right extension was significantly greater in the seated position $(p=0.003)$. No significant difference between the two groups was found in the anterior-posterior extension, in contrast to the BDW group.

\section{Correlation with Vital Capacity \& Reproducibility}

No statistically significant correlation was found between the postural change in vital capacity and the change in VuD during the breathing cycle (end-inspiration - endexpiration) in either group (healthy volunteer group: $r=0.25, p=0.28$; BDW group: $r=$ $0.60, p=0.21$; COPD group: $r=0.28, p=0.65$ ). Each participant was scanned three times in the same posture for each breathing manoeuvre. The MRI scans were reproducible within all groups, and no statistically significant within-subject variation between the identically-acquired scan sets was found, with a coefficient of variation $<10 \%$. 


\section{DISCUSSION}

A number of approaches have been developed to evaluate diaphragm function in health and disease (8). Whilst imaging approaches can reveal both anatomical and functional information, these usually require the patient to be supine, which may be poorly tolerated by patients with respiratory disease $(8,9,12)$. This study aimed to evaluate the utility of a low-field upright MRI system to develop a patient-acceptable protocol to assess the effect of posture on diaphragm morphology and function in healthy adults and subjects with potentially abnormal diaphragm function.

The main findings of this study are as follows. First, all subjects were able to undergo imaging using the upright MRI scanner. Subjects with COPD or BDW reported feeling more breathless during supine imaging, although limiting the breath-holds to a maximum of 10 seconds ensured successful scanning of all participants. The scanner has an openconfiguration, and no claustrophobia was reported.

All three groups, except for two participants with COPD, showed an increase in VuD at end-inspiration. However, the magnitude of the increase was largest in the healthy volunteers, with little difference between seated and supine. The increase in VuD at endinspiration was smaller for the BDW group in the supine position compared to the seated position and was very low for the COPD group particularly when supine. In two participants with COPD, VuD decreased at end-inspiration in the supine position. In both participants, a paradoxical decrease in left hemidiaphragm excursion and anteriorposterior extension was observed at end-inspiration in the supine position. VuD is impacted the excursion of the hemidiaphragms and the left-right and anterior-posterior extension. Hence the reduction in the left hemidiaphragm excursion and anteriorposterior extension at end-inspiration in the supine position accounts for the observed reduction in VuD. 
Looking at the excursion of the hemidiaphragms, in the healthy participants, the excursion did not differ significantly with a change in posture. This differs from a previous study (15), where diaphragmatic excursion was assessed in sitting and supine position in an upright $0.5 \mathrm{~T}$ MRI system, where a greater excursion was observed in the supine position compared to the sitting position. However, in the previous study, image acquisition was performed during free-breathing and the images were not obtained at end-inspiration and end-expiration. For analysis, a composite image was made from images at the end-inspiration phase and the end-expiration phase. Therefore, the investigators did not compare images at end-inspiration and expiration separately. Hence, this composite image analysis approach may account for the difference between the two studies.

Both patient groups showed a smaller increase in hemidiaphragm excursion at endinspiration compared with the healthy volunteers, and these differences were significantly smaller in the supine position, as with the VuD. A drop of 30-50\% in vital capacity in patients with BDW is expected on moving from upright to supine position $(9,16)$, in fact we only measured a drop of about $20 \%$ in vital capacity in our group at the time of scanning. This may suggest that there had been a partial improvement in diaphragm function in our BDW cohort since the initial diagnosis.

A different pattern of change was seen in the anterior-posterior and left-right extensions. In the healthy volunteers, the diaphragm was further extended in both directions at endinspiration in the seated position compared to the supine position. However, the diaphragm was extended to a statistically significant smaller degree in the left-right directions in the BDW group compared to the healthy volunteers in both postures, which contrasted with the COPD group, in whom a smaller extension in the anterior-posterior direction was observed in both postures. This may suggest that diaphragm fibres are affected by different mechanisms in these two groups or that different muscle fibre groups are affected. 
The similarities in diaphragm morphology and function between the two patient groups are of interest. Respiratory muscle dysfunction in COPD is well-established (17-19). Diaphragm dysfunction has been previously demonstrated (20-22), with diaphragm thinning, shortening of its fibres, and subsequent compromising of its force-generating capacity as the main characteristics of altered diaphragm morphology and function in COPD $(6,7,22)$. In our groups, the severity and similarity of overall respiratory dysfunction are further demonstrated by the similar MRC dyspnoea and SGRQ scores. Additionally, it is also possible that abnormal diaphragm movement is more severe in the COPD group than has previously been appreciated. However, this pilot study had insufficient numbers of subjects to address this issue entirely.

Furthermore, the observed diaphragm dysfunction may in part explain the rapid eye movement (REM) sleep related hypoventilation leading to desaturation and hypercapnia seen in some patients with COPD or BDW $(16,23)$ as accessory muscles are paralysed during REM sleep.

The main limitation of this study is the small number of individuals with BDW and COPD with hyperinflation. Although vital capacity in upright and supine position is the commonest pulmonary function test used to assess diaphragm function, sniff nasal inspiratory pressure and maximum inspiratory pressure are other frequently used tests $(8,24,25)$, which were not available in the present study. Additionally, some abnormal diaphragm motion may only be seen during free-breathing, rather than at end-inspiration or end-expiration. The 0.5T Paramed scanner used does not have cardio-respiratory gating and images obtained during free-breathing are severely degraded due to motion artefact. Hence, in this study we concentrated on analysing images at end-inspiration and end-expiration.

Improved diaphragm mechanics have been reported in COPD following endobronchial valve insertion $(26,27)$. Plethysmography and CT have shown increased diaphragm length and force-generating capacity following lung volume reduction surgery (28-30), all of which contribute to improved quality of life in patients. Hence, it would be of interest to study diaphragm function and morphology using MR pre- and post-volume reduction procedure. It may also shed light on cases where patients continue to report 
feeling of shortness of breath post-intervention, in whom diaphragm function may have remained impaired and account for the symptoms.

Additionally, this MRI system may be used in patients with diaphragm palsy who are commenced on non-invasive ventilation (NIV) to assess the change in diaphragm morphology overtime with NIV or lack thereof. Similarly, in selected cases with BDW who undergo diaphragmatic plication for symptomatic improvements, MR imaging can be used to target the worst affected side to maximise the improvement.

In summary, in this pilot study, we have demonstrated that it is feasible to use a combination of upright and supine MRI in an open-configuration system to assess the impact of posture on diaphragm morphology and function in patients with diaphragm weakness and those with COPD with hyperinflation. Patients tolerated scanning in this scanner, in particular for the upright image acquisition, suggesting this configuration may have a useful role to play in assessing diaphragm structure and function in a range of respiratory and other condition. 


\section{REFERENCES}

1. Fayssoil A, Behin A, Ogna A, Mompoint D, Amthor H, Clair B, et al. Diaphragm: Pathophysiology and Ultrasound Imaging in Neuromuscular Disorders. J Neuromuscul Dis. 2018;5(1):1-10. DOI: 10.3233/JND-170276

2. Supinski GS, Morris PE, Dhar S, Callahan LA. Diaphragm Dysfunction in Critical Illness. Chest. 2018 Apr;153(4):1040-51. DOI: 10.1016/j.chest.2017.08.1157

3. Kokatnur L, Rudrappa M. Diaphragmatic Palsy. Diseases. 2018 Mar;6(1):16-14. DOI: $10.3390 /$ diseases6010016

4. Kumar N, Folger WN, Bolton CF. Dyspnea as the Predominant Manifestation of Bilateral Phrenic Neuropathy. Mayo Clinic Proceedings. Mayo Foundation for Medical Education and Research; 2004 Nov 12;79(12):1563-5. DOI: $10.4065 / 79.12 .1563$

5. Aboussouan LS. Sleep-disordered Breathing in Neuromuscular Disease. Am J Respir Crit Care Med. 2015 May;191(9):979-89. DOI: 10.1164/rccm.2014122224 CI

6. Orozco-Levi M. Structure and function of the respiratory muscles in patients with COPD: impairment or adaptation? Eur Respir J. 2003 Nov 2;22(Supplement 46):41s-51s. DOI: 10.1183/09031936.03.00004607

7. O’Donnell DE, Webb KA, Neder JA. Lung hyperinflation in COPD: applying physiology to clinical practice. COPD Research and Practice. COPD Research and Practice; 2015 Jul 30;1(1):1-12. DOI: 10.1186/s40749-015-0008-8

8. American Thoracic Society/European Respiratory Society. ATS / ERS Statement on respiratory muscle testing. Vol. 166, American Journal of Respiratory and Critical Care Medicine. 2002.pp. 518-624. DOI: 10.1164/rccm.166.4.518

9. Dubé B-P, Dres M. Diaphragm Dysfunction: Diagnostic Approaches and Management Strategies. Journal of clinical medicine. 2016 Dec;5(12):113-26. DOI: $10.3390 / \mathrm{jcm} 5120113$

10. Paiva M, Verbanck S, Estenne M, Poncelet B, Segebarth C, Macklem PT. Mechanical implications of in vivo human diaphragm shape. J Appl Physiol. 1992 Apr;72(4):1407-12. DOI: 10.1152/jappl.1992.72.4.1407

11. Gauthier AP, Verbanck S, Estenne M, Segebarth C, Macklem PT, Paiva M. Threedimensional reconstruction of the in vivo human diaphragm shape at different lung volumes. J Appl Physiol. 1994 Feb;76(2):495-506. DOI: 10.1152/jappl.1994.76.2.495

12. Vostatek P, Novák D, Rychnovský T, Rychnovská Š. Diaphragm Postural Function Analysis Using Magnetic Resonance Imaging. Yue J, editor. PLoS ONE. Public Library of Science; 2013 Mar 14;8(3):e56724-13. DOI: 10.1371 /journal.pone.0056724 
13. Dubé B-P, Guerder A, Morelot-Panzini C, Laveneziana P. The clinical relevance of the emphysema- hyperinflated phenotype in COPD. COPD Research and Practice. COPD Research and Practice; 2016 Jan 6;2(1):1-11. DOI: 10.1186/s40749-0150017-7

14. Gibson GJ. Pulmonary hyperinflation a clinical overview. Eur Respir J. 1996 Dec 1;9(12):2640-9. DOI: 10.1183/09031936.96.09122640

15. Takazakura R, Takahashi M, Nitta N, Murata K. Diaphragmatic Motion in the Sitting and Supine Positions: Healthy Subject Study Using a Vertically Open Magnetic Resonance System. J Magn Reson Imaging. 2004 Apr 15;19:605-9. DOI: 10.1002/jmri.20051

16. Ricoy J, Rodríguez-Núñez N, Álvarez-Dobaño JM, Toubes ME, Riveiro V, Valdés L. Diaphragmatic dysfunction. Pulmonology. Sociedade Portuguesa de Pneumologia; 2019 Jun 10;25(4):223-35. DOI: 10.1016/j.pulmoe.2018.10.008

17. Barreiro E, la Puente de B, Minguella J, Corominas JM, Serrano S, Hussain SNA, et al. Oxidative Stress and Respiratory Muscle Dysfunction in Severe Chronic Obstructive Pulmonary Disease. Am J Respir Crit Care Med. 2005 May 15;171(10):1116-24. DOI: 10.1164/rccm.200407-8870C

18. Barreiro E, Bustamante V, Cejudo P, Gáldiz JB, Gea J, de Lucas P, et al. Guidelines for the Evaluation and Treatment of Muscle Dysfunction in Patients With Chronic Obstructive Pulmonary Disease. Archivos de Bronconeumología (English Edition). Elsevier Editorial Ltda; 2015 Aug;51(8):384-95. DOI: 10.1016/j.arbr.2015.04.027

19. Lee EN, Kim MJ. Meta-analysis of the Effect of a Pulmonary Rehabilitation Program on Respiratory Muscle Strength in Patients with Chronic Obstructive Pulmonary Disease. Asian Nursing Research. Elsevier; 2019 Feb 1;13(1):1-10. DOI: DOI: $10.1002 /$ pri.1766

20. Levine S, Kaiser L, Leferovich J, Tikunov B. Cellular adaptations in the diaphragm in chronic obstructive pulmonary disease. N Engl J Med. 1997 Dec 18;337(25):1799-806. DOI: 10.1056/NEJM199712183372503

21. Marin-Corral J, Minguella J, Ramirez-Sarmiento AL, Hussain SNA, Gea J, Barreiro E. Oxidised proteins and superoxide anion production in the diaphragm of severe COPD patients. Eur Respir J. 2009 May 29;33(6):1309-19. DOI: $10.1183 / 09031936.00072008$

22. Souza RMP, Cardim AB, Maia TO, Rocha LG, Bezerra SD, Marinho PÉM. Inspiratory muscle strength, diaphragmatic mobility, and body composition in chronic obstructive pulmonary disease. Physiother Res Int. 2019 Jan 18;24(2):e1766-6. DOI: $10.1002 /$ pri.1766

23. Budhiraja R, Siddiqi TA, Quan SF. Sleep Disorders in Chronic Obstructive Pulmonary Disease: Etiology, Impact, and Management. Journal of Clinical Sleep Medicine. 2015 Feb 20;11(3):259-70. DOI: 10.5664/jcsm.4540 
24. Fitting J-W. Sniff nasal inspiratory pressure: simple or too simple? Eur Respir J. 2006 May 1;27(5):881-3. DOI: 10.1183/09031936.06.00007906

25. Chaudri MB, Liu C, Watson L, Jefferson D, Kinnear WJ. Sniff nasal inspiratory pressure as a marker of respiratory function in motor neuron disease. Eur Respir J. 2000 Mar;15(3):539-42. DOI: 10.1034/j.1399-3003.2000.15.18.x

26. Pompeo E. Lung Volume Reduction Surgery for Emphysema Treatment: State-ofthe-Art and Perspectives. ISRN Pulmonology. 2014;2014(5):1-17. DOI: $10.1155 / 2014 / 418092$

27. Fiorelli A, Santoriello C, De Felice A, Ferrigno F, Carlucci A, De Ruberto E, et al. Bronchoscopic lung volume reduction with endobronchial valves for heterogeneous emphysema: long-term results. J Vis Surg. 2017 Nov 17;3:170-0. DOI: $10.21037 /$ jovs.2017.10.04

28. Cassart M, Pettiaux N, Gevenois PA, Paiva M, Estenne M. Effect of chronic hyperinflation on diaphragm length and surface area. Am J Respir Crit Care Med. 1997 Aug;156(2 Pt 1):504-8. DOI: 10.1164/ajrccm.156.2.9612089

29. Bloch KE, Li Y, Zhang J, Bingisser R, Kaplan V, Weder W, et al. Effect of surgical lung volume reduction on breathing patterns in severe pulmonary emphysema. Am J Respir Crit Care Med. 1997 Aug;156(2 Pt 1):553-60. D0I: 10.1164/ajrccm.156.2.9608031

30. Lando Y, Boiselle PM, Shade D, Furukawa S, Kuzma AM, Travaline JM, et al. Effect of lung volume reduction surgery on diaphragm length in severe chronic obstructive pulmonary disease. Am J Respir Crit Care Med. 1999 Mar;159(3):796805. DOI: $10.1164 /$ ajrccm.159.3.9804055 


\section{TABLES}

\begin{tabular}{l|cc}
\hline \multicolumn{1}{c}{ Table 1: Scan parameters } & Sagittal & Coronal \\
\hline Echo time (milliseconds) & 12 & 12 \\
Repetition time (milliseconds) & 162 or 185 & 162 \\
Flip angle (degrees) & 90 & 90 \\
Field of view (centimetre) & $36-38$ & $36-38$ \\
Matrix & $128 \times 160$ & $128 \times 160$ \\
Number of slices & $7-8$ & $7-8$ \\
Slice thickness (millimetre) & 6 & 6 \\
Inter-slice gap (millimetre) & 21 or 26 & 16 or 21 or 26 \\
\hline Half scans were used. & & \\
\hline
\end{tabular}




\begin{tabular}{|c|c|c|c|}
\hline Group & Healthy volunteers & BDW & COPD with hyperinflation \\
\hline \multicolumn{4}{|l|}{ Demographics } \\
\hline number & 20 & 6 & 5 \\
\hline Male : female & $9: 11$ & $1: 5$ & $2: 3$ \\
\hline Age (years) & $34 \pm 9.7$ & $61.3 \pm 11$ & $67 \pm 7.7$ \\
\hline \multicolumn{4}{|l|}{ Smoking status } \\
\hline smoker & 0 & 0 & 1 \\
\hline ex-smoker & $3^{*}$ & 3 & 4 \\
\hline never-smoker & 17 & 3 & 0 \\
\hline Body Mass Index & $25 \pm 5$ & $29.4 \pm 7.8$ & $24.6 \pm 2.8$ \\
\hline \multicolumn{4}{|l|}{ FEV1 } \\
\hline upright (Litre) & $3.5 \pm 0.8$ & $1.6 \pm 0.6$ & $1.4 \pm 0.6$ \\
\hline upright (\% predicted) & $98.2 \pm 12.9$ & $74.3 \pm 20$ & $53.3 \pm 9.1$ \\
\hline supine (Litre) & $3.3 \pm 0.7$ & $1.2 \pm 0.6$ & $1.2 \pm 0.6$ \\
\hline supine (\% predicted) & $92.5 \pm 10.9$ & $62 \pm 25.6$ & $47.7 \pm 10.3$ \\
\hline Postural change $\%$ & $4.1 \pm 0.8$ & $12.3 \pm 10.3$ & $5 \pm 5$ \\
\hline \multicolumn{4}{|l|}{ Vital capacity } \\
\hline upright (Litre) & $4.3 \pm 1$ & $2.1 \pm 1$ & $2.7 \pm 1$ \\
\hline upright (\% predicted) & $101 \pm 16$ & $78 \pm 35.6$ & $91 \pm 8$ \\
\hline supine (Litre) & $4.1 \pm 0.8$ & $1.6 \pm 1$ & $2 \pm 1$ \\
\hline supine (\% predicted) & $97 \pm 16$ & $58.7 \pm 35.4$ & $80 \pm 10$ \\
\hline postural change $\%$ & $3.9 \pm 4.3$ & $19.3 \pm 5.7$ & $11 \pm 8$ \\
\hline Residual volume \% & - & - & $169.7 \pm 36.7$ \\
\hline Functional residual capacity $\%$ & - & - & $142 \pm 14.7$ \\
\hline MRC dyspnoea score & $1 \pm 0$ & $2.7 \pm 0.8$ & $3 \pm 1$ \\
\hline SGRQ total score & $0 \pm 0$ & $37.3 \pm 17.4$ & $41.3 \pm 21.2$ \\
\hline \multicolumn{4}{|l|}{$\begin{array}{l}\text { visual analogue breathlessness } \\
\text { scale score }\end{array}$} \\
\hline upright & $1.2 \pm 0.4$ & $2.3 \pm 1.0$ & $1.0 \pm 0.0$ \\
\hline supine & $1.3 \pm 1.1$ & $4.5 \pm 1.9$ & $3.0 \pm 2.0$ \\
\hline \multicolumn{4}{|c|}{ The values listed are in the format mean \pm standard deviation. } \\
\hline \multicolumn{4}{|c|}{ * These three subjects all had less than two pack years of smoking history. } \\
\hline \multicolumn{4}{|c|}{$\begin{array}{l}\text { ABBREVIATIONS: BDW = bilateral diaphragm weakness, } \mathrm{COPD}=\text { chronic obstructive pulmonary disease, } \\
\text { FEV1 = forced expiratory volume in } 1 \text { second, MRC = Medical Research Council, SGRQ = St George's } \\
\text { Respiratory Questionnaire }\end{array}$} \\
\hline
\end{tabular}




\begin{tabular}{|c|c|c|c|c|c|c|c|}
\hline & & \multicolumn{2}{|c|}{ seated } & \multirow[t]{2}{*}{ p-value* } & \multicolumn{2}{|c|}{ supine } & \multirow[t]{2}{*}{ p-value* } \\
\hline & & end-inspiration & end-expiration & & end-inspiration & end-expiration & \\
\hline \multirow[t]{3}{*}{$\mathbf{V u D}^{\#}$} & Healthy & $1450 \pm 630$ & $820 \pm 300$ & $<0.0001$ & $1500 \pm 500$ & $840 \pm 290$ & $<0.0001$ \\
\hline & BDW & $1273.7 \pm 650.8$ & $837.7 \pm 244.0$ & 0.07 & $1260.5 \pm 476.0$ & $1012.8 \pm 351.9$ & 0.01 \\
\hline & COPD & $1509.0 \pm 987.6$ & $1117.7 \pm 725.3$ & 0.02 & $1351.1 \pm 584.8$ & $1330 \pm 565.6$ & 0.45 \\
\hline \multirow[t]{3}{*}{ 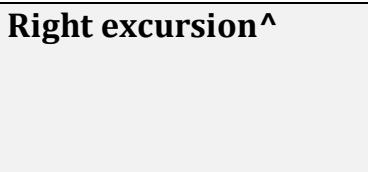 } & Healthy & $75 \pm 14$ & $541 \pm 8$ & $<0.0001$ & $72.3 \pm 9.5$ & $58.1 \pm 10.4$ & $<0.0001$ \\
\hline & BDW & $69.9 \pm 29.6$ & $60.4 \pm 14.8$ & 0.17 & $77.0 \pm 25.0$ & $73.9 \pm 18.4$ & 0.28 \\
\hline & COPD & $66.9 \pm 29.9$ & $49.9 \pm 22.4$ & 0.02 & $60.4 \pm 14.1$ & $56.9 \pm 11.8$ & 0.26 \\
\hline \multirow[t]{3}{*}{ Left excursion $^{\wedge}$} & Healthy & $64 \pm 13$ & $44 \pm 10$ & $<0.0001$ & $62.4 \pm 9.7$ & $40.8 \pm 8.0$ & $<0.0001$ \\
\hline & BDW & $61.4 \pm 27.3$ & $48.9 \pm 21.4$ & 0.15 & $56.9 \pm 26.4$ & $52.0 \pm 23.2$ & 0.17 \\
\hline & COPD & $59.3 \pm 30.7$ & $42.2 \pm 16.1$ & 0.03 & $52.4 \pm 13.1$ & $51.3 \pm 12.7$ & 0.45 \\
\hline \multirow[t]{3}{*}{ Left-Right Extension ^ } & Healthy & $240 \pm 27$ & $221.3 \pm 21.3$ & $<0.0001$ & $249.1 \pm 24.8$ & $230.1 \pm 22.2$ & $<0.0001$ \\
\hline & BDW & $227.4 \pm 202.8$ & $208.9 \pm 27.8$ & 0.02 & $232.9 \pm 35.4$ & $213.1 \pm 25.9$ & 0.02 \\
\hline & COPD & $257.9 \pm 35.0$ & $245.8 \pm 31.9$ & 0.002 & $265.6 \pm 32.4$ & $252.8 \pm 31.6$ & 0.008 \\
\hline Anterior-Posterior & Healthy & $142 \pm 22$ & $122.4 \pm 27.6$ & $<0.0001$ & $142.6 \pm 25.6$ & $125.0 \pm 20.7$ & $<0.0001$ \\
\hline \multirow[t]{2}{*}{ Extension $^{\wedge}$} & BDW & $154.0 \pm 19.4$ & $145.3 \pm 20.4$ & 0.05 & $152.8 \pm 21.7$ & $136.4 \pm 16.0$ & 0.01 \\
\hline & COPD & $168.1 \pm 34.6$ & $156.3 \pm 39.3$ & 0.02 & $157.9 \pm 31.7$ & $170.8 \pm 51.6$ & 0.29 \\
\hline \multicolumn{8}{|c|}{ The values listed are in the format mean \pm standard deviation. } \\
\hline \multicolumn{8}{|c|}{ * Paired t-test was used; the bold values denote statistical significance. } \\
\hline \multicolumn{8}{|c|}{ \# Unit of measure = millilitre } \\
\hline \multicolumn{8}{|c|}{$\wedge$ Unit of measure $=$ millimetre } \\
\hline \multicolumn{8}{|c|}{ ABBREVIATIONS: BDW = bilateral diaphragm weakness, $\mathrm{COPD}=$ chronic obstructive pulmonary disease, $\mathrm{VuD}=$ volume under the dome } \\
\hline
\end{tabular}




\begin{tabular}{|c|c|c|c|c|c|c|}
\hline Parameter & Posture & healthy & BDW & p-value ${ }^{1}$ & COPD & p-value ${ }^{2}$ \\
\hline \multirow[t]{2}{*}{ VuD \# } & \multirow{2}{*}{$\begin{array}{l}\text { seated } \\
\text { supine }\end{array}$} & $634 \pm 413$ & $436 \pm 240$ & 0.24 & $391 \pm 312$ & 0.09 \\
\hline & & $621 \pm 326$ & $248 \pm 192$ & 0.002 & $21 \pm 404$ & 0.01 \\
\hline \multirow[t]{2}{*}{ 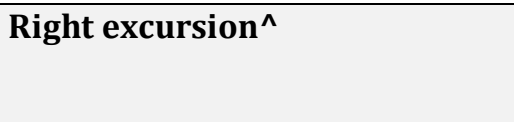 } & \multirow{2}{*}{$\begin{array}{l}\text { seated } \\
\text { supine }\end{array}$} & $21 \pm 10$ & $9 \pm 22$ & 0.14 & $17 \pm 13$ & 0.28 \\
\hline & & $14 \pm 11$ & $3 \pm 12$ & 0.04 & $3 \pm 11$ & 0.04 \\
\hline \multirow[t]{2}{*}{ 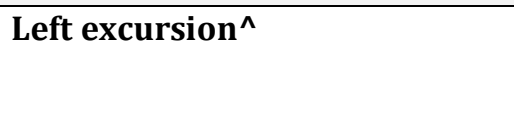 } & \multirow{2}{*}{$\begin{array}{l}\text { seated } \\
\text { supine }\end{array}$} & $21 \pm 10$ & $12 \pm 27$ & 0.26 & $17 \pm 16$ & 0.35 \\
\hline & & $20 \pm 11$ & $5 \pm 12$ & 0.007 & $1 \pm 17$ & 0.03 \\
\hline \multirow[t]{2}{*}{${\text { Left-right extension }{ }^{\wedge}}$} & \multirow{2}{*}{$\begin{array}{l}\text { seated } \\
\text { supine }\end{array}$} & $22 \pm 12$ & $18 \pm 17$ & 0.28 & $12 \pm 5$ & 0.003 \\
\hline & & $19 \pm 9$ & $20 \pm 18$ & 0.45 & $13 \pm 7$ & 0.08 \\
\hline \multirow[t]{2}{*}{ Anterior-Posterior extension ${ }^{\wedge}$} & \multirow{2}{*}{$\begin{array}{l}\text { seated } \\
\text { supine }\end{array}$} & $19 \pm 16$ & $9 \pm 11$ & 0.04 & $12 \pm 9$ & 0.11 \\
\hline & & $18 \pm 12$ & $16 \pm 12$ & 0.04 & $-13 \pm 48$ & 0.12 \\
\hline
\end{tabular}

The values listed are in the format mean \pm standard deviation.

1. Welch t-test was used to compare the healthy group with the BDW group. The bold values denote statistical significance.

2. Welch t-test was used to compare the healthy group with the COPD group. The bold values denote statistical significance.

\# Unit of measure = millilitre

^ Unit of measure $=$ millimetre

ABBREVIATIONS: BDW = bilateral diaphragm weakness, $\mathrm{COPD}=$ chronic obstructive pulmonary disease, $\mathrm{VuD}=\mathrm{volume}$ under the dome 


\section{FIGURE LEGENDS}

Figure 1 - A subject can be seen in the upright scanner position supine (A.) and seated (B.). Lines indicative of the excursion of the two hemidiaphragms and left-right and anterior-posterior extensions are drawn on coronal (C.) and sagittal (D.) plane images of a healthy volunteer. $\mathrm{VuD}$ is the volume between the diaphragm surface and a planar base surface spanning axially at the most inferior point of the diaphragm.

Figure 2 - A. Illustration of three overlaid coronal and sagittal surfaces estimated from the manually selected scattered points. B. The final average surface constructed from the individual coronal and sagittal surfaces.

Figure 3 - These are representative coronal and sagittal plane images of the three cohort. These non-contrast images were acquired on a Paramed open upright 0.5T MRI scanner. A. 34-year-old male healthy volunteer with $2 \%$ postural change in vital capacity. B. 49year-old female with BDW with 29\% postural change in vital capacity. C. 57-year-old female with COPD with hyperinflation with $23 \%$ postural change vital capacity.

Figure 4 - The change in the five parameters of diaphragm morphology with the breathing cycle in seated and supine positions is shown for each group: A. VuD, B. right hemidiaphragm excursion, C. left hemidiaphragm excursion, D. anterior-posterior extension, and E. left-right extension, The circles represent the parameter value. The black lines connect end-expiration circle in the seated position to end-inspiration circle in the seated position and end-expiration circle in the seated position to end-inspiration circle in the supine position for each participant. The grey line connects the endinspiration circle in the seated position to the end-expiration circle in the supine position. 

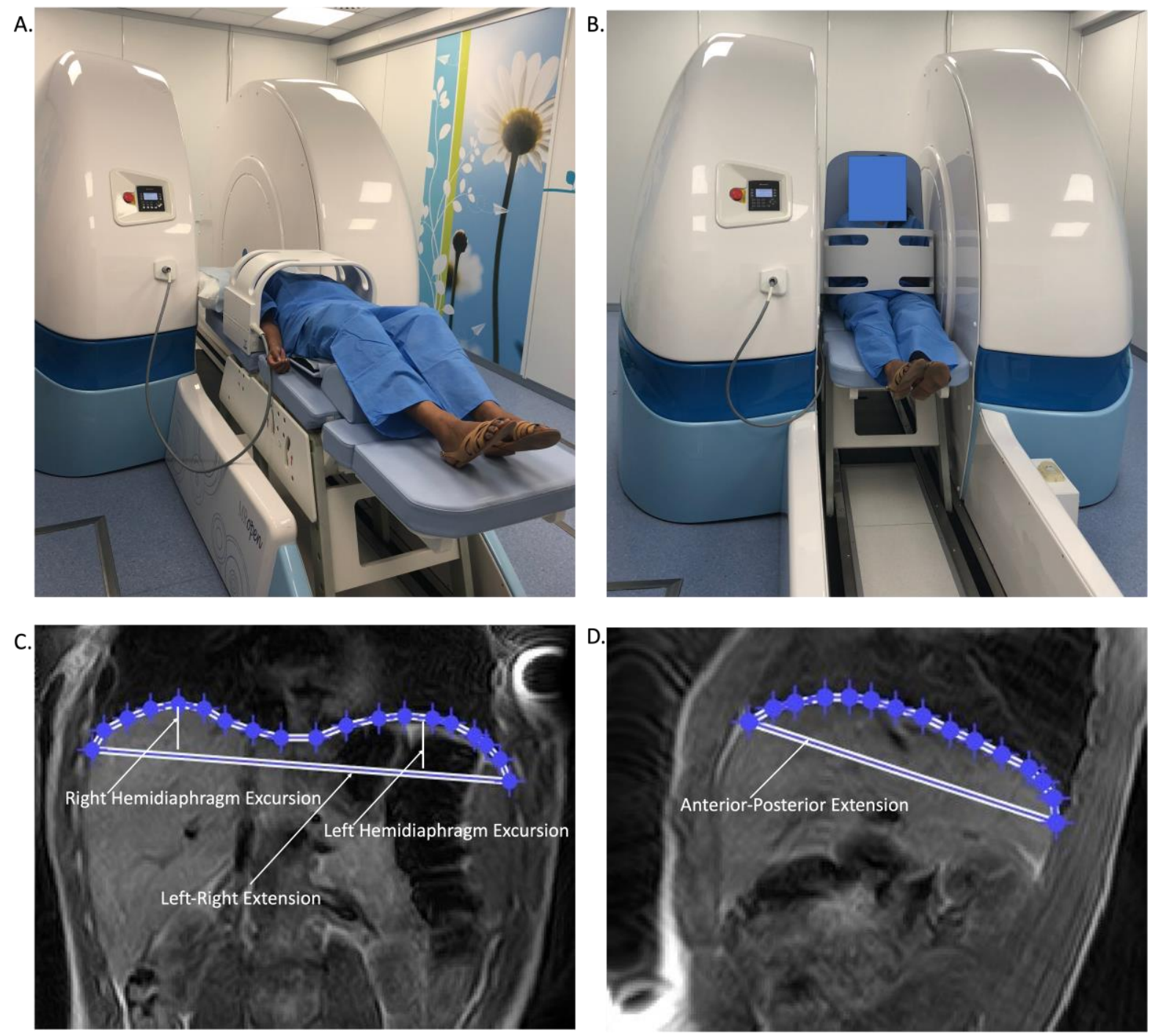

Figure 1 - A subject can be seen in the upright scanner position supine (A.) and seated (B.). Lines indicative of the excursion of the two hemidiaphragms and left-right and anterior-posterior extensions are drawn on coronal (C.) and sagittal (D.) plane images of a healthy volunteer. $\mathrm{VuD}$ is the volume between the diaphragm surface and a planar base surface spanning axially at the most inferior point of the diaphragm. 

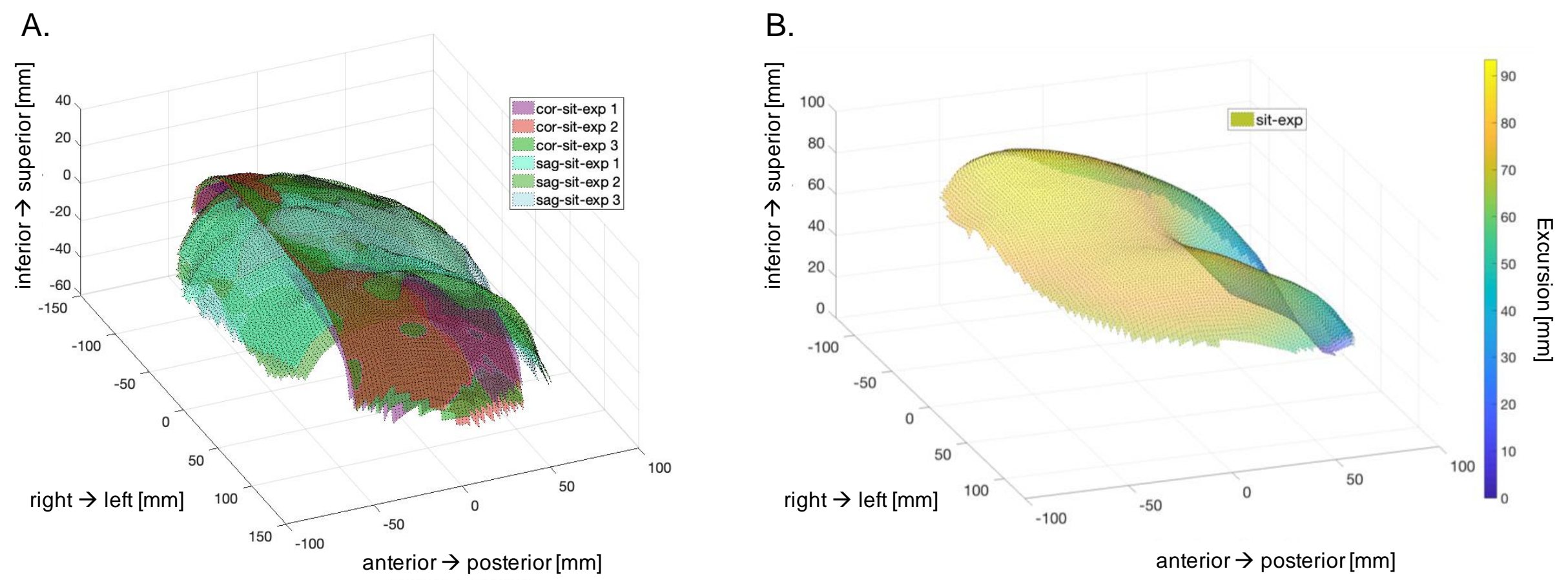

Figure 2 - These are representative composite coronal and sagittal images of a healthy participant at end-expiration in the sitting position.

A. Illustration of three overlaid coronal and sagittal surfaces estimated from the manually selected scattered points. B. The final average surface constructed from the individual coronal and sagittal surfaces.

ABBREVIATIONS: cor $=$ coronal, $\mathrm{mm}=$ millimetre, $\mathrm{sag}=$ sagittal, sit-exp $=$ sitting at end-expiration 


\section{Coronal}

sitting

expiration

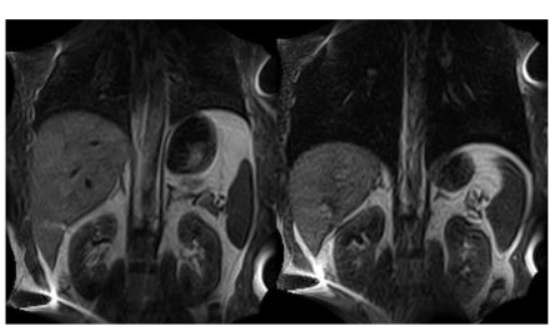

A.

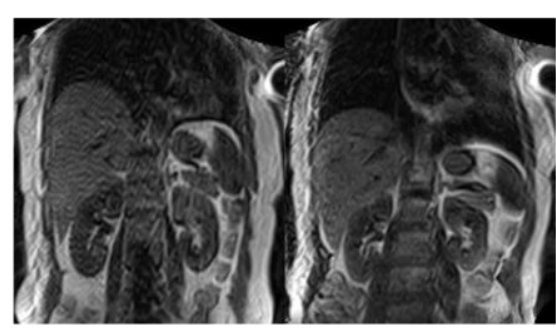

c.

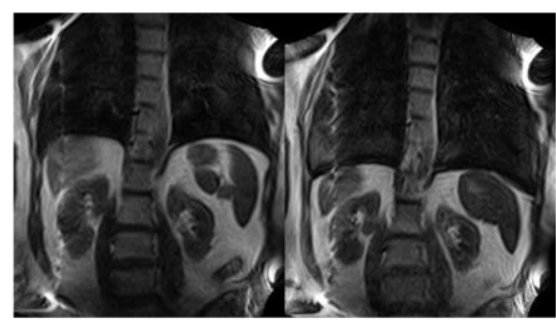

supine
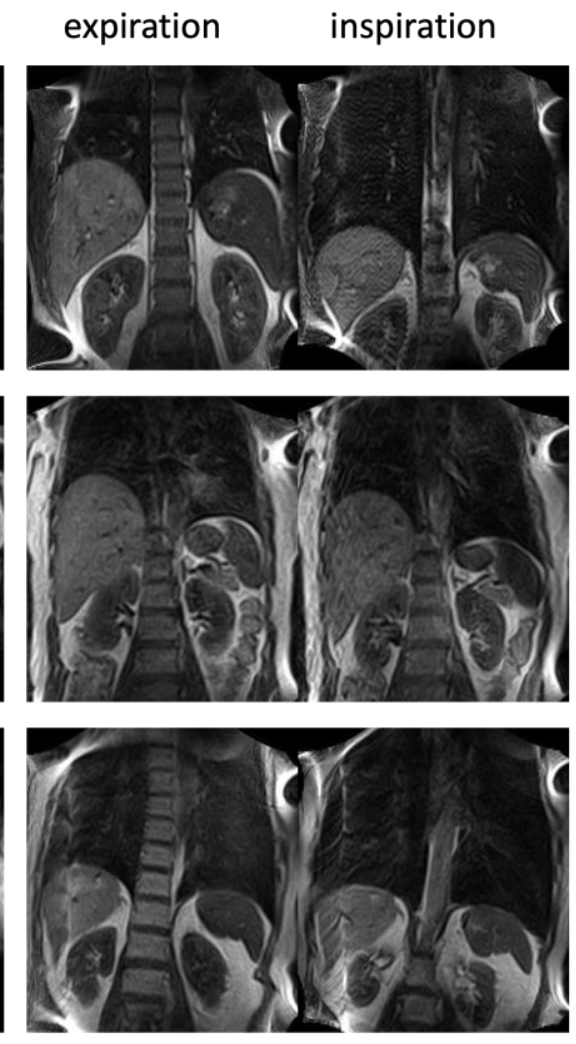

Sagittal
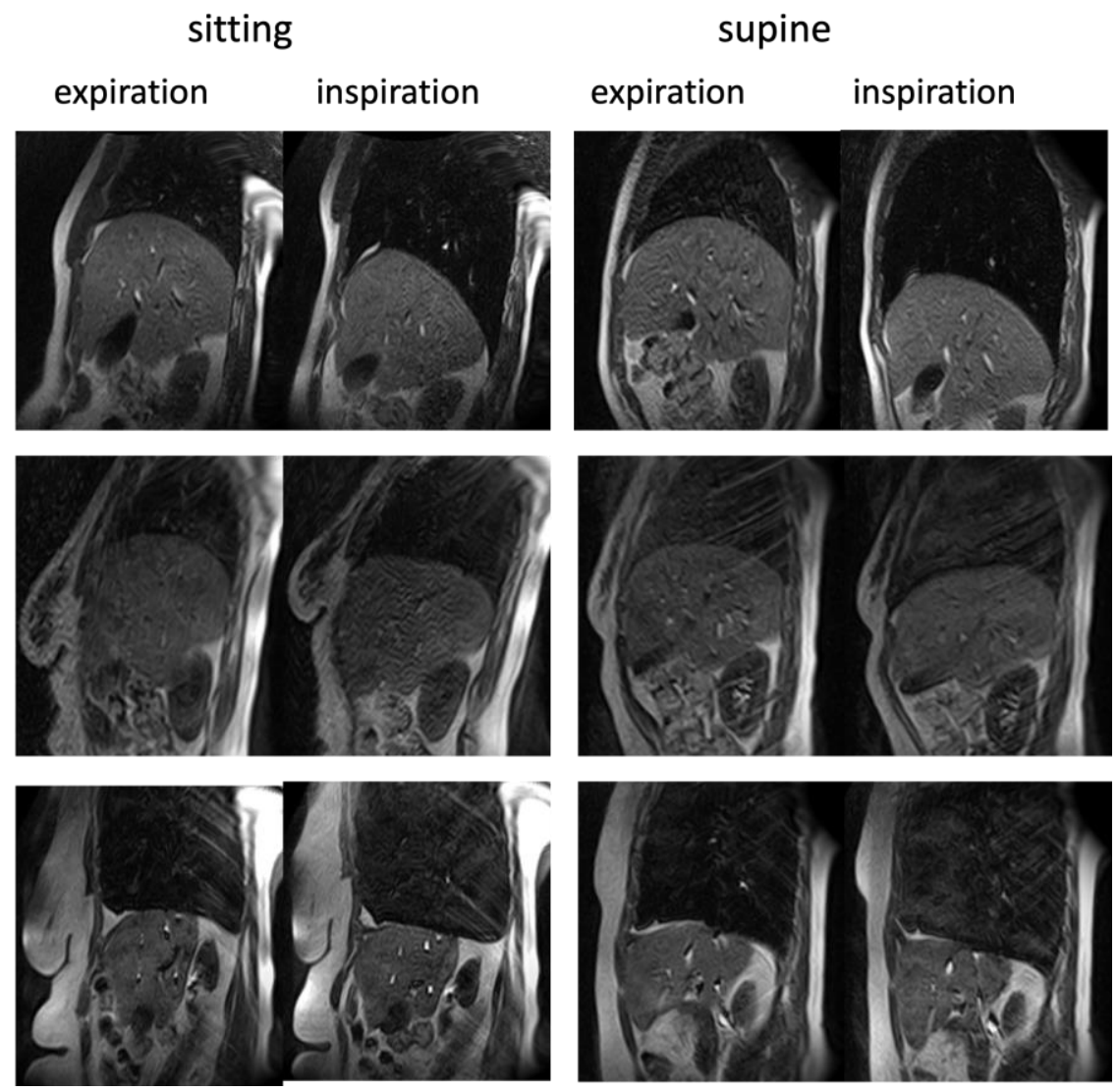

Figure 3 - These are representative coronal and sagittal plane images of the three cohort. These non-contrast images were acquired on a Paramed open upright 0.5T MRI scanner. A. 34-year-old male healthy volunteer with 2\% postural change in vital capacity. B. 49-year-old female with BDW with 29\% postural change in vital capacity. C. 57-year-old female with COPD with hyperinflation with $23 \%$ postural change vital capacity. 

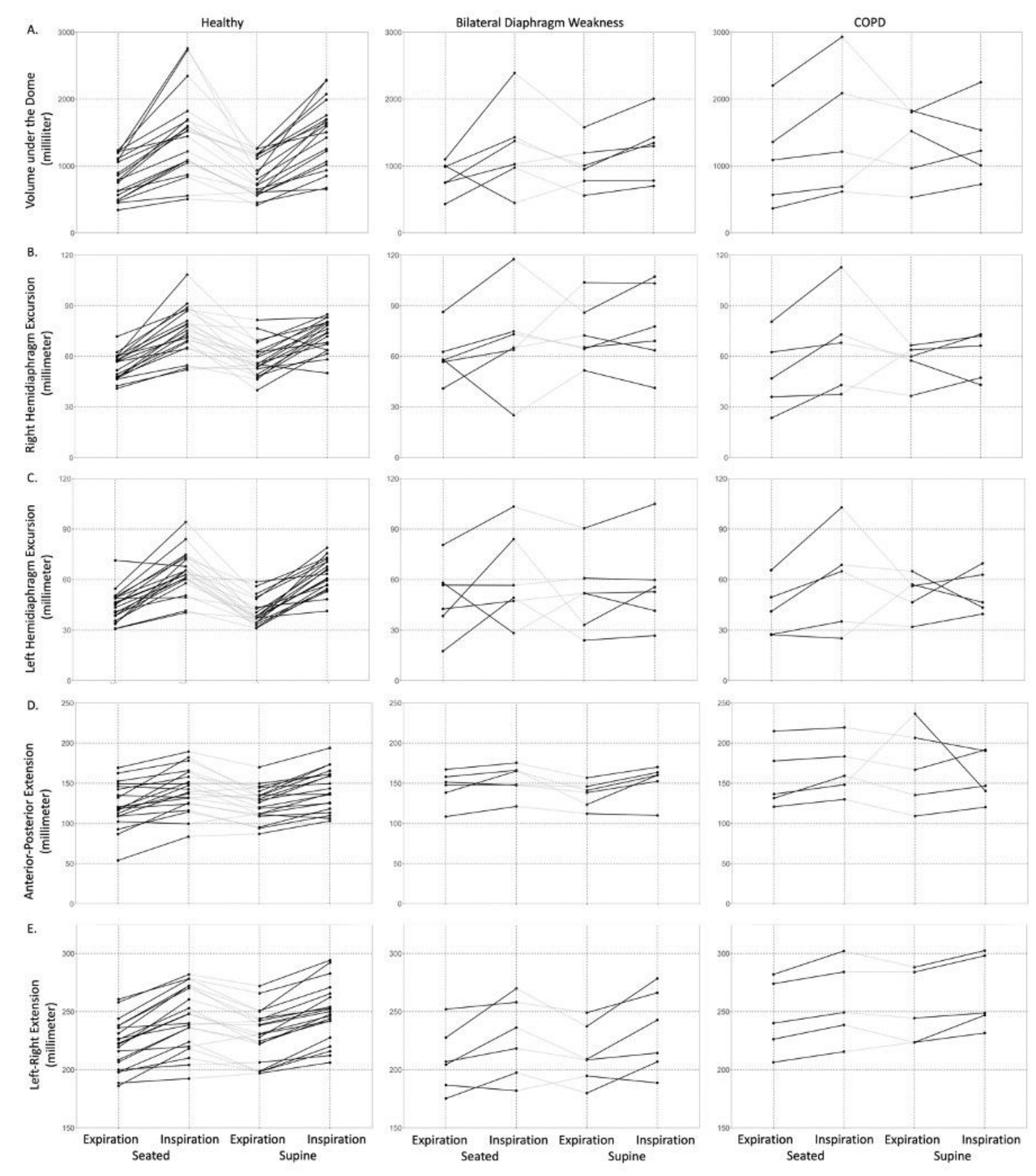

Figure 4 - The change in the five parameters of diaphragm morphology with the breathing cycle in seated and supine positions is shown for each group: A. VuD, B. right hemidiaphragm excursion, C. left hemidiaphragm excursion, D. anterior-posterior extension, and E. left-right extension, The circles represent the parameter value. The black lines connect end-expiration circle in the seated position to end-inspiration circle in the seated position and end-expiration circle in the seated position to end-inspiration 
circle in the supine position for each participant. The grey line connects the endinspiration circle in the seated position to the end-expiration circle in the supine position. 